\title{
Enhanced Backscattering for Dielectrically Active Scatterers
}

\author{
Ari Sihvola, Rashda Parveen, Henrik Wallén, and Pasi Ylä-Oijala
}

Abstract - The scattering characteristics of dielectrically active spheres of arbitrary electrical size are discussed. Using electromagnetic Lorenz-Mie analysis, differences in the scattering behavior of dissipative, lossless, perfect-electric-conductor, and active scattering objects are highlighted. The focus is on the enhanced backscattering effect of an active object compared to a dissipative or lossless one.

\section{Introduction}

Scattering by dielectric spheres is a classical problem in electromagnetics. Due to the groundbreaking early studies by Ludvig Valentin Lorenz [1] and Gustav Mie [2], the machinery of computing scattering, absorption, and extinction of penetrable spheres is generally called Lorenz-Mie scattering. This scattering theory has made it possible to analyze a large variety of phenomena, like rainbow color details, attenuation by clouds, radar cross sections of spherical targets, and modeling of materials on the micro and nano levels. With the rapid advances in computing power in recent decades, Mie scattering expansions can be effectively coded and used to find extremely accurate predictions even for large scatterers. Pedagogically excellent textbooks on Lorenz-Mie scattering have been published, like the ones by Hendrik C. van de Hulst [3] and Craig F. Bohren and Donald R. Huffman [4].

Mie scattering analysis has been extended to layered spheres [5], impedance-surface spheres [6], and certain other complex spherical scatterers [7]. However, it seems that it has not very much been applied to active scatterers - in other words, spheres whose constitutive material is characterized by gain (for example, spheres made of lasing media, where the gain results from stimulated emission of photons with the use of a pump source). There are sporadic publications from the late 1970 s on Mie scattering by active spheres (by N. G. Alexopoulos [8] and M. Kerker [9, 10], and a recent treatment of Kerker scattering conditions [11]). However, considering the significance of active electromagnetic materials in the present-day metamaterials scene,

Manuscript received 21 December 2021.

Ari Sihvola, Henrik Wallén, and Pasi Ylä-Oijala are with the Department of Electronics and Nanoengineering, School of Electrical Engineering, Aalto University, P.O. Box 15500, 00076 AALTO, Espoo, Finland; e-mail: ari.sihvola@aalto.fi, henrik. wallen@aalto.fi,pasi.yla-oijala@aalto.fi.

Rashda Parveen was with the Department of Electronics and Nanoengineering, School of Electrical Engineering, Aalto University, Espoo, Finland as a visiting researcher. Presently she is with the Department of Electronics, Quaid-i-Azam University, Islamabad, Pakistan; e-mail: rashda@ele.qau.edu.pk. this problem has not received due attention, which is one of the reasons for the present contribution.

In this article, we will revisit the problem of when an electromagnetic wave interacts with an active scatterer. Using the Lorenz-Mie formalism for the scattering, absorption, and extinction cross sections of spheres, we focus of the scattering behavior of dielectric, isotropic, homogeneous active spheres. In particular, we point out, by numerical examples and novel results, the enhanced backscattering character of active spheres.

\section{Mie Coefficients and Active Scatterers}

The scattering, extinction, and absorption efficiencies of a sphere are [4]

$$
\begin{gathered}
Q_{\text {sca }}=\frac{2}{x^{2}} \sum_{n=1}^{\infty}(2 n+1)\left(\left|a_{n}\right|^{2}+\left|b_{n}\right|^{2}\right) \\
Q_{\text {ext }}=\frac{2}{x^{2}} \sum_{n=1}^{\infty}(2 n+1) \operatorname{Re}\left\{a_{n}+b_{n}\right\} \\
Q_{\text {abs }}=Q_{\text {ext }}-Q_{\text {sca }}
\end{gathered}
$$

where the electric and magnetic Mie coefficients are

$$
\begin{aligned}
& a_{n}=\frac{\sqrt{\varepsilon} \psi_{n}(m x) \psi_{n}^{\prime}(x)-\sqrt{\mu} \psi_{n}(x) \psi_{n}^{\prime}(m x)}{\sqrt{\varepsilon} \psi_{n}(m x) \xi_{n}^{\prime}(x)-\sqrt{\mu} \xi_{n}(x) \psi_{n}^{\prime}(m x)} \\
& b_{n}=\frac{\sqrt{\mu} \psi_{n}(m x) \psi_{n}^{\prime}(x)-\sqrt{\varepsilon} \psi_{n}(x) \psi_{n}^{\prime}(m x)}{\sqrt{\mu} \psi_{n}(m x) \xi_{n}^{\prime}(x)-\sqrt{\varepsilon} \xi_{n}(x) \psi_{n}^{\prime}(m x)}
\end{aligned}
$$

for a sphere with relative permittivity $\varepsilon$, relative permeability $\mu$, refractive index $m=\sqrt{\varepsilon \mu}$, and size parameter $x=2 \pi a / \lambda$, where $a$ is the radius of the sphere and $\lambda$ the free space wavelength. Here, the RiccatiBessel functions $\psi_{n}$ and $\xi_{n}$ are defined as

$$
\psi_{n}(\rho)=\rho j_{n}(\rho), \quad \xi_{n}(\rho)=\rho h_{n}^{(2)}(\rho)
$$

where $j_{n}$ and $h_{n}$ are the usual spherical Bessel and Hankel functions. For a perfect-electric-conductor (PEC) sphere, we have $\varepsilon \rightarrow \infty, \mu \rightarrow 0$, and $m$ can be assumed finite; and the Mie coefficients (4) simplify to

$$
a_{n}=\frac{\psi_{n}^{\prime}(x)}{\xi_{n}^{\prime}(x)}, \quad b_{n}=\frac{\psi_{n}(x)}{\xi_{n}(x)}
$$

In the following, we focus on dielectric scatterers - in other words, spheres with a relative permeability of $\mu=1$. Then the sign of the imaginary part of the relative permittivity $\varepsilon$ determines whether the scatterer 


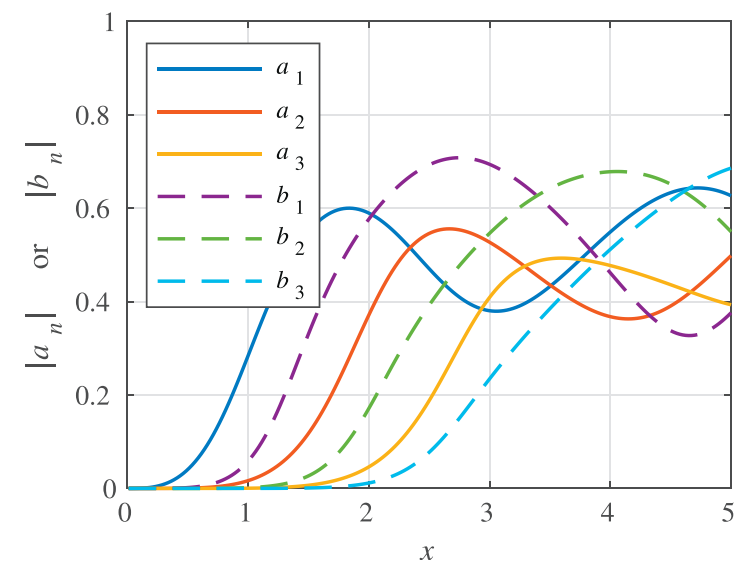

Figure 1. Behavior of the absolute value of Mie coefficients - the first three electric $\left(a_{1}, a_{2}, a_{3}\right)$ and magnetic $\left(b_{1}, b_{2}, b_{3}\right)$ multipoles - as a function of the size parameter $x$ for a dissipative scatterer having $\varepsilon^{\prime}=$ $3, \varepsilon^{\prime \prime}=1.5$.

is active or passive. We use the notation for timeharmonic waves as follows: $\exp (\mathrm{j} \omega t)$. Therefore the Hankel functions appearing in the Mie coefficients are of the second kind $\left(h_{n}^{(2)}\right)$.

With this notation, the complex expansion of the permittivity is written as $\varepsilon=\varepsilon^{\prime}-\mathrm{j} \varepsilon^{\prime \prime}$. Hence dissipative materials have $\varepsilon^{\prime \prime}>0$, whereas $\varepsilon^{\prime \prime}<0$ corresponds to active media. If $\varepsilon^{\prime \prime}=0$, the medium is lossless. Following common terminology, both dissipative and lossless materials are considered passive.

A straightforward computation of the lowest-order Mie coefficients reveals the radically different character of active and passive spheres. Figures 1 and 2 show the behavior of the magnitudes of electric $\left(a_{1}, a_{2}, a_{3}\right)$ and magnetic $\left(b_{1}, b_{2}, b_{3}\right)$ Mie coefficients. The active and passive spheres in this numerical study have mirrorimage permittivities - in other words, the only difference between them is that the imaginary part is swapped: $\varepsilon=3-\mathrm{j} 1.5$ and $\varepsilon=3+\mathrm{j} 1.5$. While the coefficients of the passive sphere in Figure 1 are limited

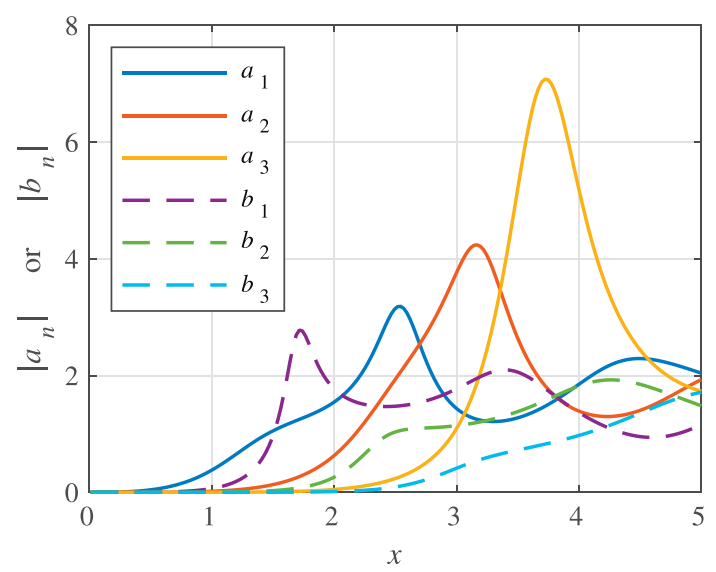

Figure 2. Absolute value of Mie coefficients as in Figure 1 for an active scatterer with $\varepsilon^{\prime}=3, \varepsilon^{\prime \prime}=-1.5$, again as a function of the size parameter $x$.

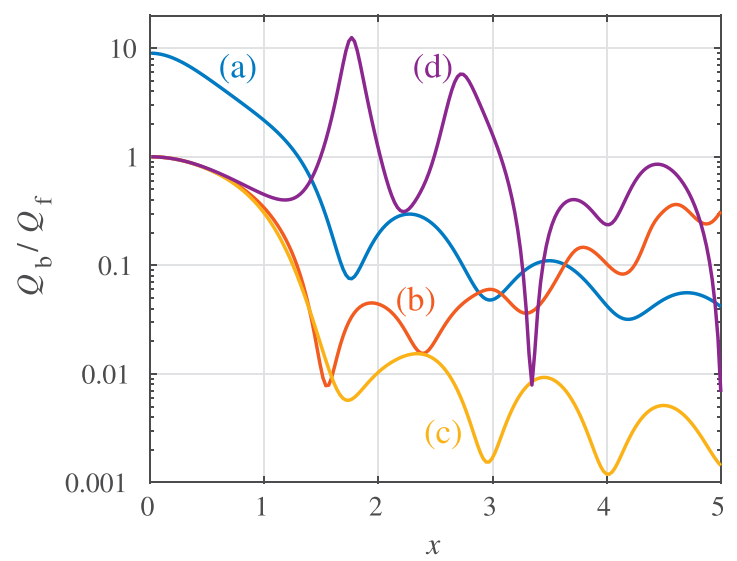

Figure 3. The back-to-front scattering ratio (in logarithmic scale) by spheres of different character, as a function of the size parameter $x$ : (a) perfect electric conductor, (b) lossless, $\varepsilon^{\prime}=3, \varepsilon^{\prime \prime}=0$, (c) dissipative, $\varepsilon^{\prime}$ $=3, \varepsilon^{\prime \prime}=1.5$, (d) active, $\varepsilon^{\prime}=3, \varepsilon^{\prime \prime}=-1.5$.

in amplitude to values below unity, it is seen that they can reach very high magnitudes in the active scattering case in Figure 2.

\section{Backscattering Enhancement}

A particularly interesting effect in connection with active spheres is the fact that in the scattering process, they divert very much of the scattered energy in the backward direction, compared with lossless and passive spheres. In the following, we quantify this effect with numerical computations. In other words, we look at the scattering patterns and compute and compare how much of the scattered power goes into the backward and forward hemispheres. This we do using two figures of merit: the ratio of back-to-front scattering of the sphere, and the so-called asymmetry parameter. Both quantities provide interesting information about the scattering behavior of the sphere.

The backscattering efficiency of a sphere is [4]

$$
Q_{\mathrm{b}}=\frac{1}{x^{2}}\left|\sum_{n=1}^{\infty}(2 n+1)(-1)^{n}\left(a_{n}-b_{n}\right)\right|^{2}
$$

Using the scattering amplitude in the forward direction [4], we can similarly define the forward-scattering efficiency as

$$
Q_{\mathrm{f}}=\frac{1}{x^{2}}\left|\sum_{n=1}^{\infty}(2 n+1)\left(a_{n}+b_{n}\right)\right|^{2}
$$

Figure 3 shows the back-to-front scattering ratio $Q_{\mathrm{b}} / Q_{\mathrm{f}}$ as function of the size parameter $x$, for four types of sphere: dissipative, lossless, active, and PEC. This parameter behaves very differently in the four cases.

As is known [12], a PEC sphere which is very small compared to the wavelength has a ninefold backscattering cross section compared to forward scattering. This is visible and conspicuous in Figure 3, 


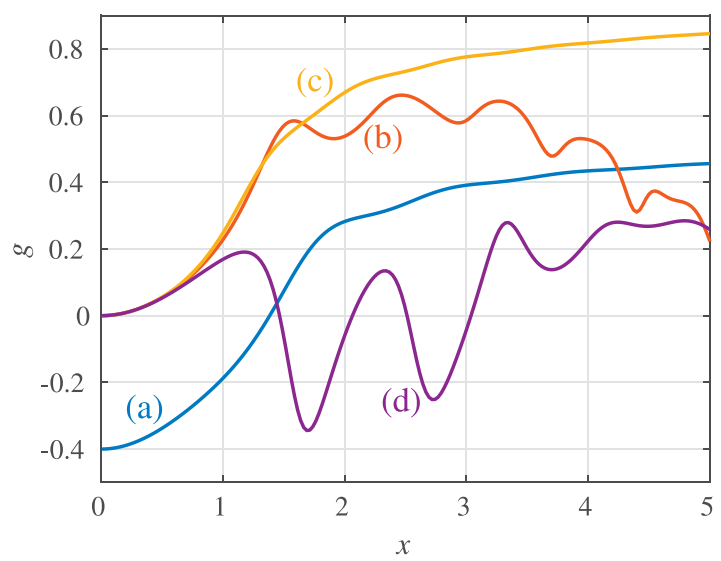

Figure 4. The asymmetry parameter $g$ for spheres of different character, as a function of the size parameter $x$ : (a) perfect electric conductor, (b) lossless, $\varepsilon^{\prime}=3, \varepsilon^{\prime \prime}=0$, (c) dissipative, $\varepsilon^{\prime}=3, \varepsilon^{\prime \prime}=1.5$, (d) active, $\varepsilon^{\prime}=3, \varepsilon^{\prime \prime}=-1.5$.

as well as the fact that when the electrical size of the PEC sphere increases, forward scattering starts to dominate. In contrast, in all three dielectric cases the back-to-front ratio is unity for small spheres. When the size increases and the diameter of the sphere becomes comparable with the wavelength or overtakes it, this ratio varies in an irregular oscillating manner. However, a particularly interesting fact worth noting is that the $Q_{\mathrm{b}} / Q_{\mathrm{f}}$ ratio for the case of an active sphere can reach rather high values for large spheres (in this particular case, it may exceed 10, whereas the other scatterers have a clear preference for forward scattering).

The other figure of merit in how the scatterer diverts the incoming radiation into the spatial directions is the asymmetry parameter $g$. Using the Mie coefficients (4), this asymmetry parameter can be computed in the following way [4]:

$$
g=\langle\cos \theta\rangle=\frac{Q_{\mathrm{sca}}\langle\cos \theta\rangle}{Q_{\text {sca }}}
$$

where

$$
\begin{aligned}
Q_{\text {sca }}\langle\cos \theta\rangle= & \frac{4}{x^{2}} \sum_{n=1}^{\infty} \frac{n(n+2)}{n+1} \operatorname{Re}\left\{a_{n} a_{n+1}^{*}+b_{n} b_{n+1}^{*}\right\} \\
& +\frac{4}{x^{2}} \sum_{n=1}^{\infty} \frac{2 n+1}{n(n+1)} \operatorname{Re}\left\{a_{n} b_{n}^{*}\right\}
\end{aligned}
$$

This parameter takes into account the scattering not only in the exact scattering angles $0^{\circ}$ and $180^{\circ}$ but averaged with the $\cos \theta$ weight over the whole solid angle.

Using this asymmetry parameter $g$, we arrive at another view of the scattering behavior of different spherical scatterers. Figure 4 displays $g$ for the same four spheres that were treated in Figure 3, again as function of the electrical size $x$. Unlike the back-to-front scattering ratio, this parameter is limited to values between -1 and +1 . Positive values correspond to scatterers for which scattering in the forward directions dominates, where negative values tell that the direct or oblique backscattering is stronger.

The information from Figure 4 is similar to that from Figure 3. The low-frequency $(x \ll 1)$ deviation of the PEC spheres from dielectric ones appears clearly. Another observation is that the oscillations in $Q_{\mathrm{b}} / Q_{\mathrm{f}}$ curves are damped when illustrated in the $g(x)$ functions. Nevertheless, the basic message of the two illustrations is the same: active scatterers tend to scatter much more in backward directions compared to passive scatterers.

\section{Conclusion}

The emphasis in this article has been on the scattering behavior of active objects (isotropic, dielectric, homogeneous, spherical objects with negative imaginary part $\varepsilon^{\prime \prime}$ of the permittivity). Such scatterers have a radically stronger tendency to scatter in the backward half-space compared to lossless and dissipative dielectric spheres. It is worth reiterating here that we have benchmarked and reconfirmed previous analytical Mie scattering computations against surface-integral-equation-based computations. This opens an avenue to extending the study: investigating, using numerical methods, how the geometrical shape of the scatterer affects this backscattering-enhancement phenomenon.

\section{References}

1. L. V. Lorenz, "Lysbevægelser i og uden for en af plane Lysbølger belyst Kugle," Kongelige Danske Videnskabernes Selskabs Skrifter, 6, 6, 1890, pp. 2-62.

2. G. Mie, "Beiträge zur Optik trüber Medien, speziell kolloidaler Metallösungen," Annalen der Physik, 330, 3, 1908, pp. 377-445.

3. H. C. van de Hulst, Light Scattering by Small Particles, New York, Dover, 1981.

4. C. R. Bohren and D. R. Huffman, Absorption and Scattering of Light by Small Particles, New York, Wiley, 1983.

5. A. L. Aden and M. Kerker, "Scattering of Electromagnetic Waves From Two Concentric Spheres," Journal of Applied Physics, 22, 10, October 1951, pp. 1242-1246.

6. A. Sihvola, B. Kong, P. Ylä-Oijala, D. C. Tzarouchis, and H. Wallén, "Scattering, Extinction, and Albedo of Impedance-Boundary Objects," URSI Radio Science Letters, 1, 2019, doi: 10.46620/19-0009.

7. H. Wallén, I. V. Lindell, and A. Sihvola, "MixedImpedance Boundary Conditions," IEEE Transactions on Antennas and Propagation, 59, 5, May 2011, pp. 15801586.

8. N. G. Alexopoulos and N. K. Uzunoglu, "Electromagnetic Scattering From Active Objects: Invisible Scatterers," Applied Optics, 17, 2, January 1978, pp. 235-239.

9. M. Kerker, "Electromagnetic Scattering From Active Objects," Applied Optics, 17, 21, November 1978, pp. 3337-3339.

10. M. Kerker, "Resonances in Electromagnetic Scattering by Objects With Negative Absorption," Applied Optics, 18, 8, April 1979, pp. 1180-1189. 
11. J. Olmos-Trigo, C. Sanz-Fernández, D. R. Abujetas, J. Lasa-Alonso, N. de Sousa, et al., "Kerker Conditions Upon Lossless, Absorption, and Optical Gain Regimes," Physical Review Letters, 125, August 2020, p. 073205 .
12. A. Sihvola, P. Ylä-Oijala, and I. V. Lindell, "Scattering by PEMC (Perfect Electromagnetic Conductor) Spheres Using Surface Integral Equation Approach, Applied Computational Electromagnetics Society Journal, 22, 2, July 2007, pp. 236-249. 